\title{
Stability Analysis for a Self-exciting Mechanism with Friction Using Interval Computations
}

\author{
Sébastien Berger ${ }^{\mathrm{a}}$ and Jean Jacques Sinou ${ }^{\mathrm{b}}$ \\ ${ }^{a}$ Laboratoire Modélisation Intelligence Processus Systèmes- EA 2332, \\ Ecole Nationale Supérieure d'Ingénieurs Sud Alsace, \\ 12 rue des frères Lumière, 68093 Mulhouse Cedex, France. \\ Email: sebastien.berger@uha.fr \\ ${ }^{b}$ Laboratoire de Tribologie et Dynamique des Systèmes UMR 5513, \\ Ecole Centrale de Lyon,36 avenue Guy de Collongue, 69134 Ecully, France. \\ Corresponding Author, Email: jean-jacques.sinou@ec-lyon.fr
}

\begin{abstract}
:
The aim of this paper is to present a new strategy based on the interval analysis for the prediction of stable and unstable regions of mechanical systems subjected to friction-induced instability. For the sake of simplicity, a twodegree-of-freedom minimal model is constructed and analysed to examine the numerical procedure and interval algorithm. The efficiency and robustness of the proposed method is demonstrated through numerical simulations versus the variation of the friction coefficient and the damping factor.
\end{abstract}

\section{KEYWORDS:}

Self-exciting mechanism; Stability analysis; Interval analysis; Friction-induced vibration; Robust design.

\section{CITATION:}

S. Berger and J.J. Sinou. 2011. Stability analysis for a self-exciting mechanism with friction using interval computations, Int. J. Vehicle Structures \& Systems, 3(1), 14-20.

\section{Introduction}

In the field of engineering, friction-induced vibrations are a major concern in a wide variety of mechanical systems. In recent decades, the subject of frictioninduced vibration has received considerable attention from a number of researchers [1-4]. Their investigations were conducted in order to find different mechanisms of friction-induced system instability. This type was presented in the context of brake noise to predict the dynamic behaviour of brake systems and to prevent instability [5-9]. Such friction-induced self-generated vibrations can be explained by four general, independent mechanisms, namely stick-slip, speed dependent friction force, sprag-slip and mode coupling [1, 2, 10-19]. The former two rely on tribological properties whereas the latter two are due to geometrical conditions. Interested readers may refer to papers $[1,2,10]$ for an overview of the four general mechanisms.

Though many studies have been conducted and some of them have been successfully applied to particular systems and running conditions, it will be very difficult to find suitable values of the system parameters in order to obtain stable systems for all operating conditions. In the study of dynamical systems depending on a given control parameter, the engineer therefore needs to conduct stability analysis which is the first important point in instability phenomena and allows him to find suitable devices to characterize and to design a mechanical system. Thanks to an increase in computer capabilities, a parametric analysis on various physical parameters is the most common solution for defining suitable design guidelines to predict noise generation and its dependency on system parameters. However, parametric studies can still be extremely time-consuming and costly to perform in terms of storage requirements. This paper presents a new methodology for predicting stable and unstable regions versus variation of physical parameters. The principal idea is to reduce the number of parametric studies by performing an interval analysis.

In the first part of the paper, the modal coupling mechanism used in this study and the classic stability analysis will be briefly presented. Secondly, some basic concepts about interval arithmetic will be described, as well as the strategy for predicting instability phenomena. The paper outlines the stability analysis and gives a strategy by constructing the stable and unstable regions based on the interval analysis and the Routh-Hurwitz criterion. Finally, performance and suitability of this approach are tested in the case of a simple mechanical system with two degrees of freedom (DoF). The results obtained are compared with those estimated in a classic deterministic parametric study through numerical simulations corresponding to different physical configurations.

\section{Mechanical Model Description}

The mechanical system under study, shown in Fig. 1, is composed of a mass $m$ held against a moving band. It is assumed that the mass and band surfaces are always in contact. This assumption may be due to a preload 
applied to the system. The contact between the mass and the band is modelled by two plates supported by two different springs. The friction coefficient at contact is assumed to be constant and the band moves at a constant velocity. Then it is assumed that the direction of friction force does not change because the relative velocity between the band speed and $\dot{X}_{1}$ or $\dot{X}_{2}$ is assumed to be positive. This simple theoretical two DoF system, first presented by Hultén [20, 21] and resumed by Sinou and L. Jézéquel [16], has been chosen as the mechanical model, but it does not intend to capture all the geometrical properties of any real system with friction interfaces. It helps to investigate friction-induced vibrations via analytical developments and then to demonstrate efficiency of the proposed methodology to find the stability boundaries with the interval analysis into a numerical algorithm using the interval analysis.

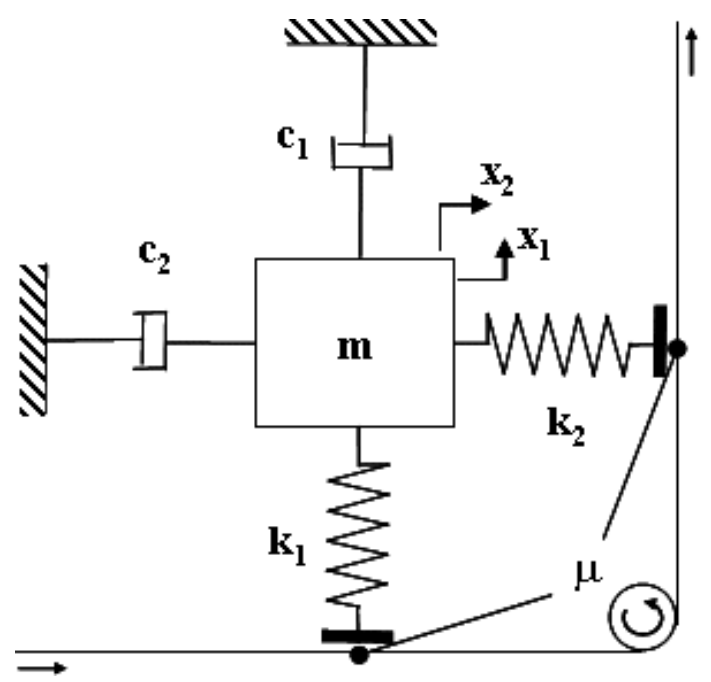

Fig. 1: Mechanical system

Concerning the friction forces between the two plates and the band, Coulomb's law, $F_{T}=\mu F_{N}$, is assumed. Where $F_{T}$ is the tangential force and $F_{N}$ is the normal force. The equations of motion assuming $F_{N}$ to be relating linearly to displacement of the mass normal to the contact surface can be expressed as:

\section{$\mathbf{M} \ddot{\mathbf{X}}+\mathbf{C} \dot{\mathbf{X}}+\mathbf{K X}=\mathbf{0}$.}

Where $\mathbf{X}=\left(\begin{array}{ll}X_{1} & X_{2}\end{array}\right)^{T} . \ddot{\mathbf{X}}, \dot{\mathbf{X}}$ and $\mathbf{X}$ are the acceleration, velocity, and displacement response, 2dimensional vectors of the DoF. The mass matrix $\mathbf{M}$, the damping matrix $\mathbf{C}$, and the stiffness matrix $\mathbf{K}$ are given by:

$$
\begin{aligned}
& \mathbf{M}=\left[\begin{array}{ll}
m & 0 \\
0 & m
\end{array}\right] \\
& \mathbf{C}=\left[\begin{array}{ll}
c_{1} & 0 \\
0 & c_{2}
\end{array}\right] \\
& \mathbf{K}=\left[\begin{array}{cc}
k_{1} & -\mu k_{2} \\
\mu k_{1} & k_{2}
\end{array}\right]
\end{aligned}
$$

$\mathbf{K}$ is asymmetric as a result of the friction force. Therefore, this system may become unstable. By dividing these equations by $m$ and considering the relative damping coefficients, $\eta_{i}=c_{i} / \sqrt{m_{i} k_{i}},(i=1,2)$ and natural pulsations, $\omega_{0, i}=\sqrt{k_{i} / m_{i}},(i=1,2)$, the following relations can be established:

$$
\begin{gathered}
{\left[\begin{array}{ll}
1 & 0 \\
0 & 1
\end{array}\right]\left(\begin{array}{l}
\ddot{X}_{1} \\
\ddot{X}_{2}
\end{array}\right)+\left[\begin{array}{cc}
\eta_{1} \omega_{0,1} & 0 \\
0 & \eta_{2} \omega_{0,2}
\end{array}\right]\left(\begin{array}{l}
\dot{X}_{1} \\
\dot{X}_{2}
\end{array}\right)} \\
+\left[\begin{array}{cc}
\omega_{0,1}^{2} & -\mu \omega_{0,2}^{2} \\
\mu \omega_{0,1}^{2} & \omega_{0,2}^{2}
\end{array}\right]\left(\begin{array}{l}
X_{1} \\
X_{2}
\end{array}\right)=\left(\begin{array}{l}
0 \\
0
\end{array}\right)
\end{gathered}
$$

The base physical parameters are given in Table 1 .

Table 1: Deterministic value of the physical parameters [16]

\begin{tabular}{lc}
\hline Description & Value \\
\hline First natural pulsation $\left(\omega_{0,1}\right)$ & $1000 / 2 \pi \mathrm{Hz}$ \\
Second natural pulsation $\left(\omega_{0,2}\right)$ & $800 / 2 \pi \mathrm{Hz}$ \\
Mass $(m)$ & $1 \mathrm{~kg}$ \\
First relative damping coefficient $\left(\eta_{1}\right)$ & 0.02 \\
Second relative damping coefficient $\left(\eta_{2}\right)$ & 0.02 \\
Friction coefficient $(\mu)$ & 0.3 \\
\hline
\end{tabular}

\section{Stability Analysis}

The stability of the static solution is investigated by calculating the Routh-Hurwitz criterion. The goal of the following section is to develop the analytical expressions of the Routh-Hurwitz coefficients versus the deterministic parameters which govern the stability of the static solution of the considered mechanical system. Considering Eqn. (5):

$$
\begin{aligned}
& \operatorname{det}\left(\lambda^{2} \mathbf{M}+\lambda \mathbf{C}+\mathbf{K}\right)= \\
& \quad\left|\begin{array}{cc}
\lambda^{2}+\eta_{1} \omega_{0,1} \lambda+\omega_{0,1}^{2} & -\mu \omega_{0,2}^{2} \\
\mu \omega_{0,1}^{2} & \lambda^{2}+\eta_{2} \omega_{0,2} \lambda+\omega_{0,2}^{2}
\end{array}\right|
\end{aligned}
$$

The $4^{\text {th }}$-order characteristic polynomial can be obtained:

$$
\lambda^{4}+a_{1} \lambda^{3}+a_{2} \lambda^{2}+a_{3} \lambda+a_{4}=0
$$

where $\lambda$ are the eigenvalues of the linearized mechanical system. The expressions $a_{1}, a_{2}, a_{3}$ and $a_{4}$ are given by:

$$
\begin{aligned}
& a_{1}=\eta_{1} \omega_{0,1}+\eta_{2} \omega_{0,2} \\
& a_{2}=\eta_{1} \eta_{2} \omega_{0,1} \omega_{0,2}+\omega_{0,1}^{2}+\omega_{0,2}^{2} \\
& a_{3}=\eta_{1} \omega_{0,1} \omega_{0,2}^{2}+\eta_{2} \omega_{0,2} \omega_{0,1}^{2} \\
& a_{4}=\omega_{0,1}^{2} \omega_{0,2}^{2}\left(1+\mu^{2}\right)
\end{aligned}
$$

The Routh-Hurwitz coefficients are then defined by the following expressions:

$$
\begin{aligned}
& H_{1}=a_{1} \\
& H_{2}=a_{1} a_{2}-a_{3}
\end{aligned}
$$




$$
\begin{aligned}
& H_{3}=a_{1} a_{2} a_{3}-a_{3}^{2}-a_{4} a_{1}^{2} \\
& H_{4}=a_{1} a_{2} a_{3} a_{4}
\end{aligned}
$$

If all these coefficients are positive, the static solution of the mechanical system is stable. When at least one of the coefficients $H_{i} \quad$ (with $i=1, \ldots, 4$ ) is negative, this static solution is unstable. For the reader's comprehension, the stability of the static solution can also be investigated by calculating the eigenvalues of the system which can be found by solving the characteristic Eqn. (6); as long as the real part of all the eigenvalues remains negative, the static solution of the system is stable. When at least one of the eigenvalues has a positive real part, the static solution is unstable. The imaginary part of this eigenvalue represents the frequency of the unstable mode.

Considering the fourth previous expression, it may be observed that,

$$
\begin{aligned}
H_{1}= & \eta_{1} \omega_{0,1}+\eta_{2} \omega_{0,2}>0 \\
H_{2}= & \eta_{1} \omega_{0,1}^{3}+\eta_{2} \omega_{0,2}^{3} \\
& +\eta_{1} \eta_{2} \omega_{0,1} \omega_{0,2}\left(\eta_{1} \omega_{0,1}+\eta_{2} \omega_{0,2}\right)>0 \\
H_{4}= & \omega_{0,1}^{4} \omega_{0,2}^{4}\left(\eta_{1} \omega_{0,1}+\eta_{2} \omega_{0,2}\right) \\
& \left(\eta_{1} \eta_{2}+\omega_{0,1} / \omega_{0,2}+\omega_{0,2} / \omega_{0,1}\right) \\
& \left(\eta_{1} \omega_{0,2}+\eta_{2} \omega_{0,1}\right)\left(1+\mu^{2}\right)>0
\end{aligned}
$$

Then, the stability of the static solution is governed only by the Routh-Hurwitz coefficients $\mathrm{H}_{3}$,

$$
\begin{aligned}
H_{3} & =\omega_{0,1}^{2} \omega_{0,2}^{2}\left(( \eta _ { 1 } \omega _ { 0 , 1 } + \eta _ { 2 } \omega _ { 0 , 2 } ) \left(\eta_{1} \eta_{2}+\omega_{0,1} / \omega_{0,2}\right.\right. \\
& \left.+\omega_{0,2} / \omega_{0,1}\right)\left(\eta_{1} \omega_{0,2}+\eta_{2} \omega_{0,1}\right)-\left(\eta_{1} \omega_{0,2}+\eta_{2} \omega_{0,1}\right)^{2} \\
& \left.-\left(1+\mu^{2}\right)\left(\eta_{1} \omega_{0,1}+\eta_{2} \omega_{0,2}\right)^{2}\right)
\end{aligned}
$$

Fig. 2(a) shows the evolution of the Routh-Hurwitz coefficient $H_{3}$ for various values of the friction coefficient $\mu$ and various damping $\eta_{1}$ while keeping the structural damping $\eta_{2}$ at its base value $\left(\eta_{2}=0.06\right)$. The boundary between the stable and unstable zones is given by using Eqn. (19).

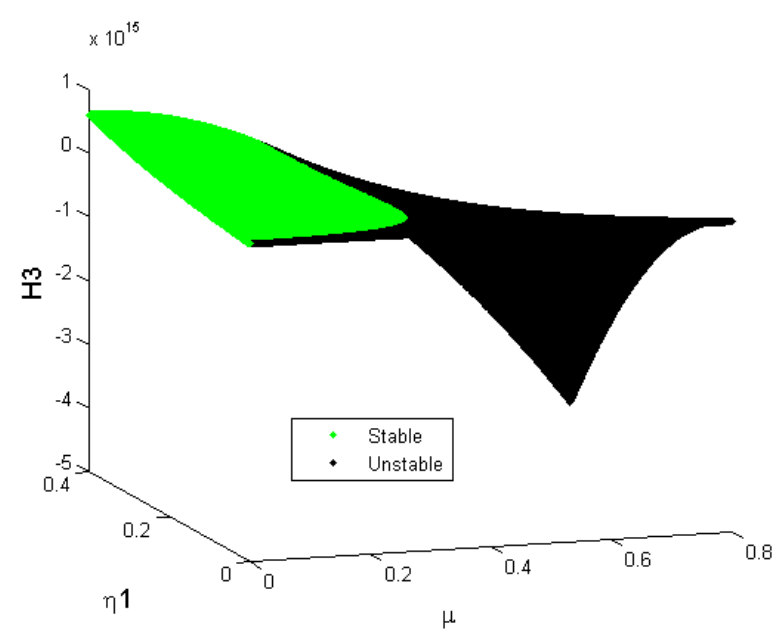

Fig. 2(a): Deterministic study: Routh-Hurwitz coefficient $\mathbf{H}_{3}$
Fig. 2(b) shows these stable and unstable areas. As previously explained by Hoffmann and Gaul [22], Sinou and Jézéquel [16], and Kirillov [23] [24], if nonproportional damping is introduced, the mechanical system may become more unstable.

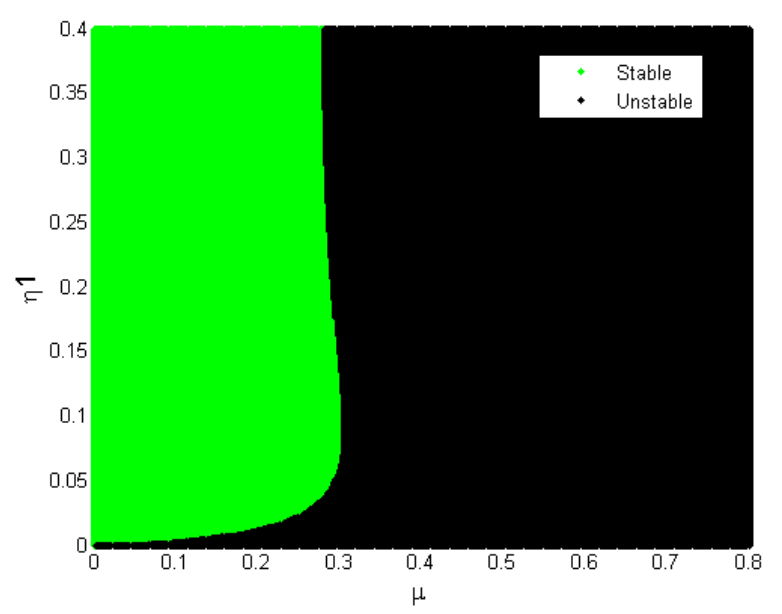

Fig. 2(b): Deterministic study: Stability map

\section{Interval Approach}

After a brief presentation of the interval theory with its advantages and disadvantages, the exploration strategy of the design space is described. Then, three stability studies of the friction system based on the interval arithmetic are presented.

\subsection{Interval Theory}

An interval is a subset of the reals $R$ [25] [26]. It is defined as:

$$
[\mathrm{x}]=\{\mathrm{t} / \underline{\mathrm{x}} \leq \mathrm{t} \leq \overline{\mathrm{x}}, \underline{\mathrm{x}} \text { and } \overline{\mathrm{x}} \in \mathrm{R}\}
$$

An interval is denoted in brackets with its two bounds

$$
[\mathrm{x}]=\lfloor\underline{\mathrm{x}}, \overline{\mathrm{x}}\rfloor
$$

An interval arithmetic is developed. Thus, it is possible to implement directly several basic operations on intervals such as addition, subtraction, multiplication, division as follows:

$$
\begin{aligned}
& {[\mathrm{x}]+[\mathrm{y}]=\lfloor\underline{x}+\underline{\mathrm{y}}, \overline{\mathrm{x}}+\overline{\mathrm{y}}\rfloor} \\
& {[\mathrm{x}]-[\mathrm{y}]=\lfloor\underline{\mathrm{x}}-\overline{\mathrm{y}}, \overline{\mathrm{x}}+\underline{\mathrm{y}}\rfloor} \\
& {[\mathrm{x}] *[\mathrm{y}]=\lfloor\min (\underline{\mathrm{x}} \underline{\mathrm{y}}, \overline{\mathrm{x}} \overline{\mathrm{y}}, \overline{\mathrm{x}} \underline{\mathrm{y}}, \overline{\mathrm{x}} \overline{\mathrm{y}}), \max (\underline{\mathrm{x}} \underline{\mathrm{y}}, \underline{\mathrm{x}} \overline{\mathrm{y}}, \overline{\mathrm{x}} \underline{\mathrm{y}}, \overline{\mathrm{x}} \overline{\mathrm{y}})\rfloor(24)} \\
& 1 /[\mathrm{x}]=\lfloor 1 / \overline{\mathrm{x}}, 1 / \underline{\mathrm{x}}\rfloor \quad(0 \notin[\mathrm{x}]) \\
& {[\mathrm{x}] /[\mathrm{y}]=[\mathrm{x}] *(1 /[\mathrm{y}]) \quad(0 \notin[\mathrm{y}])}
\end{aligned}
$$

However, there is no function to determine the system eigenvalues. It is necessary to improve the methods based on the operations which can be developed with the interval arithmetic. The major advantage of the interval arithmetic is to work directly with intervals. Nevertheless, this approach has disadvantages. The simple application of interval arithmetic which consists in replacing all arithmetic operations and the functions by their equivalents in intervals can lead to significant over-estimates. These over-estimates can be due to several phenomena. 
First, interval calculation considers that each occurrence in an equation or an equation system of a parameter modeled by an interval is independent. Indeed, for example, with a subtraction $[a]-[b]$, the interval algebra gives, as a result, the interval [c] which runs any element of $[a]$ minus any element of $[b]$. This independence of the occurrences, called pessimism, does not correspond to the physical reality of the studied system. Thus, in the case of a coefficient [c1] modeled by the interval [1,2], the subtraction does not give a zero interval, but the interval $[-1,1]$ as,

$$
\left[\mathrm{c}_{1}\right]=[1,2]-[1,2]=[-1,1] \neq[0,0]
$$

With an interval of length one, a simple subtraction leads to an interval of length two. This pessimism due to multiple occurrences of the same uncertainty leads to an overestimation of the real solution.

Second, interval arithmetic cannot correctly represent complex fields of the state variables in the state space, while using interval boxes whose sides are parallel to the axes. For example, the interval calculation algorithm considers that the image of an interval is a single interval, which is not always the case. It is possible that the image is made up of various distinct zones, whereas the solution found is a single interval including all these various zones. In this case, the wrapping effect gives an interval which over-estimates the exact image in an excessive way. Finally, according to the form under which the state equations are written, the intervals representing the state variables can be different. The amplitudes of the intervals can vary significant. So, the form under which the state equations are written is of primary significance.

In order to limit the pessimism phenomenon, various occurrences of the parameters and the state variables were factorized in the equations as often as possible. When it is not possible to factorize the parameters in certain components of the equations, some studies of functions can be carried out. One way to limit the "wrapping effect" is the sub-pavement. It consists in subdividing the original set into different areas, and studying the behaviour of the system in each of these areas. By cutting the original area fairly thin, it is possible to significantly reduce the overestimation and thus obtain narrower intervals [27] [28] [29]. So, in the present study, the method of sub-paving will be applied from a cutting strategy which will be explained in the following section. To reduce the overestimation of the amplitudes of intervals, Jaulin proposed the idea to add additional equations to those of the model [27].

These additional equations, called contractors, are always checked as mathematical relationships. For example, with an angle $\theta$ the contractor can be the following equation:

$$
\cos ^{2} \theta+\sin ^{2} \theta=1
$$

The model equations give a range for the angle $\theta$, which is introduced into the contractor to obtain a new range for $\theta$. The intersection between these two intervals can define a closer interval for $\theta$. This process can be repeated iteratively until convergence to an interval. However, in this study, no contractor can be defined.

\subsection{Strategy}

In a classic parametric deterministic stability study, the design space must be discretized. However, the choice of the step size is difficult and arbitrary. Nothing justifies the choice and gives the certainty to obtain a stability map in adequacy with the real behaviour of the system. In order to identify all instability areas the designer must choose a very thin step, which leads to numerous calculations and calculation time becomes prohibitive. Furthermore, between two discretization points, the stability state is not known. The interval arithmetic can relieve these two drawbacks. Indeed, the main interest of interval arithmetic is the possibility to study the system stability, not on a single point of the design space, but on an area of the design space which includes a set of design points. Thereby, the stability state of an area can be determined in one time with absolute certainty.

To determine the stability state of a given area of the design space defined by intervals for each parameter, the Routh-Hurwitz criteria are calculated with the interval arithmetic. Thus, the system is stable if all intervals of the Routh-Hurwitz criteria are positive. The following three cases are possible:

1. All intervals are positive, so the area is stable,

2. At least one interval is negative, so the area is unstable,

3. At least one interval holds both positive values and negative values while the other intervals are positive, so the stability state cannot be determined. There is uncertainty.

The following two reasons may account for this uncertainty:

1. There are both unstable points and stable points in the studied area, and

2. The stability state is the same all over the area. However, the pessimism and the wrapping effect lead to overestimation of the real solution.

Therefore, it is necessary to build a cutting strategy of the design space with areas which are small enough in order to avoid the overestimation effect and wide enough to decrease the number of calculations. The strategy is shown in Fig. 3. The method is based on two steps:

- Discretization of the design space with wide areas to allow few calculations, and

- Cutting the area whose stability is uncertain to adjust the area size near the boundary between the stable and unstable zones with a stopping criterion based on a minimum area not to be exceeded.

The stopping criterion is defined as follows: the lengths of all dimensions of the area for each parameter must be lower than given limits. For each parameter, these limits can be different. For example, in a biparametric study, if the stability state is uncertain, the area is cut into 4 subzones (into 2 for each parameter) whose stability state is studied again. This process which uses a recursive function is repeated until the stability state is certain or the area size becomes lower than a given limit. If the stability state is uncertain and the stopping criterion is not reached, the stability map is not built but the calculation is counted in the number of calculations. 


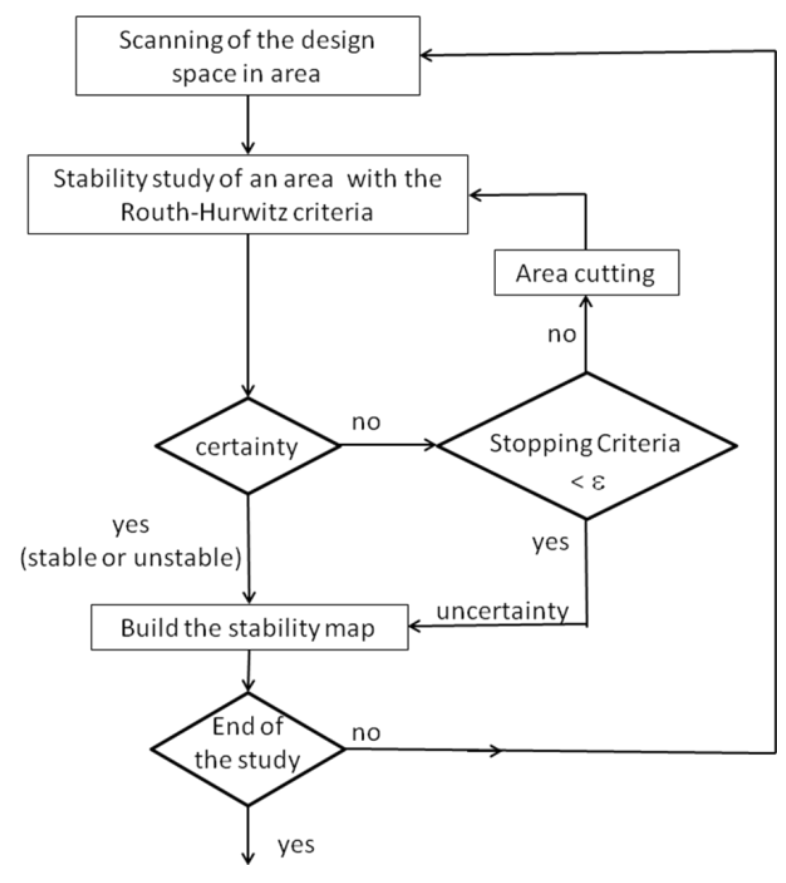

Fig. 3: Cutting strategy

\subsection{Stability Analysis}

Three stability analyses of the friction system based on the interval arithmetic are presented. The calculations were performed with the Matlab toolbox called Intlab. The studies were conducted according to two parameters: the friction coefficient $\mu$ and the first relative damping coefficient $\eta_{l}$. However, this method may be generalized to a larger number of parameters. The classic deterministic biparametric analysis presented in Section 3 being the reference study, the design space will always be the same.

A first study based on the cutting strategy of the design space described in the previous Section 3 is shown. The discretization steps are respectively 0.4 for $\mu$ and 0.008 for $\eta_{l}$. The stopping criteria are equal to the discretization steps of the classic deterministic study presented in Section 3: 0.002 for $\mu$ and 0.001 for $\eta_{l}$. Fig. 4 shows the stability map according to $\mu$ and $\eta_{l}$. The stability state is given in the middle of each studied area. The results correlate with the deterministic study. A very small area of uncertainty appears between the stable and unstable areas. The studied areas are wide (lengths equal to 0.2 for $\mu$ and 0.004 for $\eta_{l}$ ) far from the boundary between the stable and unstable zones and much smaller near the boundary. Indeed, as described in Section 4.2, the study of an area stops when the lengths of all dimensions of the area are below the given limits.

When the stopping criterion on $\mu$ is reached (0.002), the length for $\eta_{1}$ is equal to 0.0000625 . So, the benefit in the number of calculations made with the use of wide areas near the boundary is mitigated by very small areas near the boundary. Finally, the number of calculations (119304) is lower by about $25 \%$ than that obtained for the deterministic study (160 081). Thus, the interval approach is well suited for the stability studies of areas far from the boundary between the stable and unstable zones. However, the interval approach becomes less effective near the boundary because of the overestimation effect which requires cutting areas excessively. In fig. 3, the lower and upper limits of the interval of the coefficient $H_{3}$ are plotted in the middle of each studied area. All coefficient values determined with the deterministic approach are always included in the intervals obtained with the interval approach.

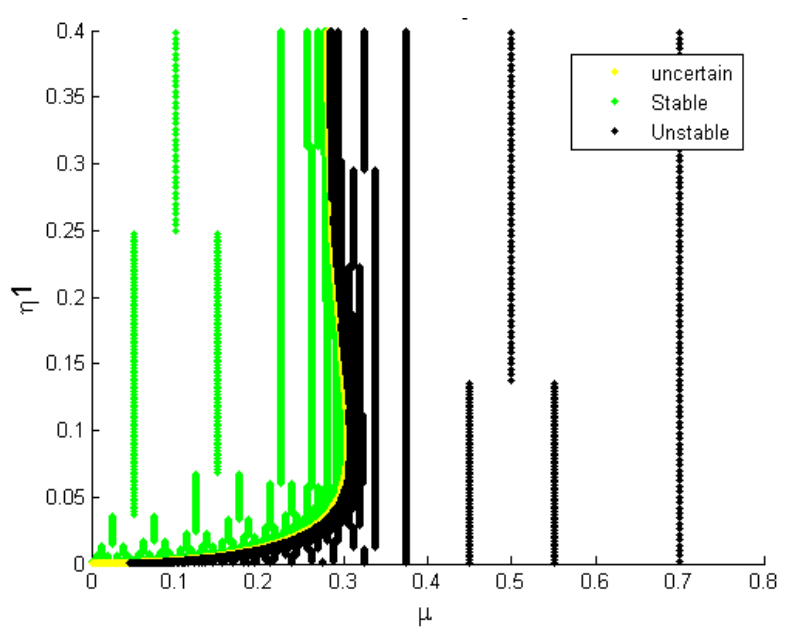

Fig. 4: Accurate interval study: Stability map

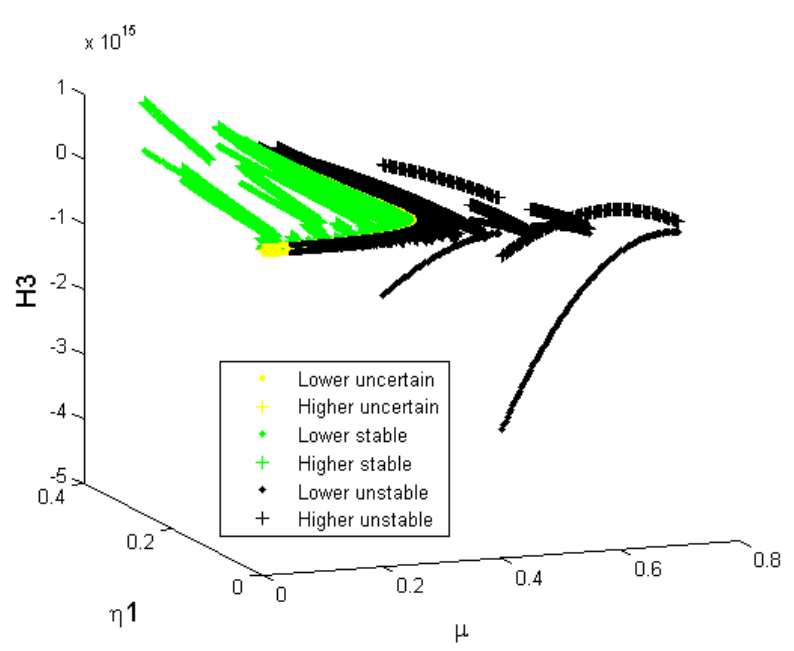

Fig. 5: Accurate interval study: Routh-Hurwitz coefficient H3

The idea of the second study is to use higher stopping criteria to drastically reduce the number of calculations so that the uncertain zone does not increase significantly. A study similar to the previous one is carried out with the same stopping criterion for $\eta_{1}$, but ten times greater for $\mu$ (0.02 instead of 0.002). Figs. 6 and 7 show respectively the stability map and the evolution of the Routh-Hurwitz coefficient $\mathrm{H}_{3}$ according to the friction coefficient $\mu$ and the damping $\eta_{1}$. The number of calculations (13180) decreases sharply. It is twelve times smaller than that obtained for the deterministic study (160081). This corresponds approximately to a decrease of $91 \%$. The uncertain area increases but remains limited. Therefore, not to explore the uncertain area helps to limit the number of calculations significantly.

The objective of the third study is to use the advantages of the interval approach for the boundary and, at the same time, avoid the disadvantages which occur near the boundary with the deterministic approach. 


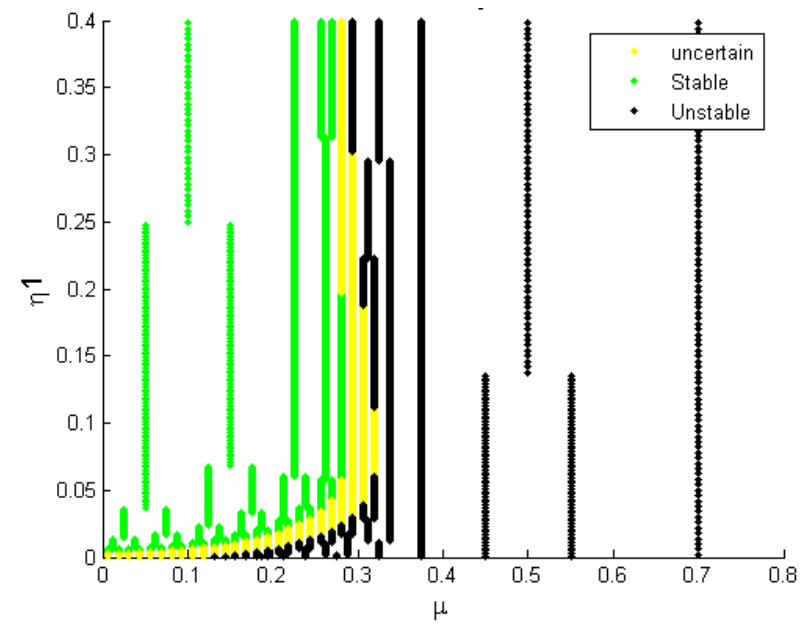

Fig. 6: Rough interval study: Stability map

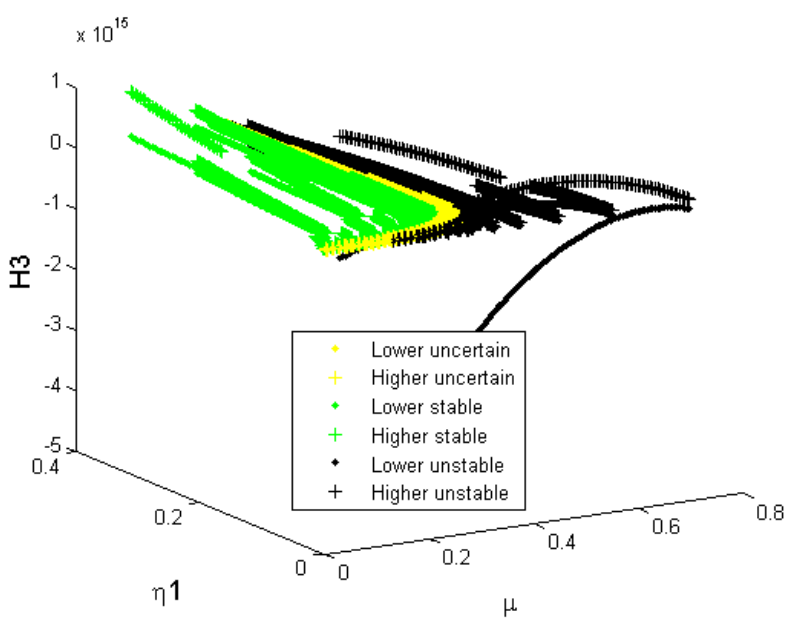

Fig. 7: Rough interval study: Routh-Hurwitz coefficient $\boldsymbol{H}_{3}$

The third study is divided into two stages:

1. Interval approach using soft stopping criteria to obtain a stability map with few calculations but with a significant uncertain area, and

2. Exploration of uncertain areas with a classic parametric study to refine the determination of the boundary without too many calculations.

In the first stage, the second study shown above (Figs. 6 and 7) is performed. In the second stage, a classic deterministic parametric study is carried out about the areas of uncertainty with the same discretization step as the reference study. So, a stability map is built (Fig. 8). There are no areas of uncertainty. Moreover, the number of calculations is low (see Table 2); they amount to 20931 distributed as follows: 13180 for the interval approach and 7751 for the deterministic study. This approximately corresponds to a decrease of $91 \%$ compared to the reference study. There are two possibilities for designers:

- Only stage 1 is performed. A stability map is built with a low number of calculations. The stable areas are $100 \%$ certain. There is no risk of forgetting any unstable points. The areas of uncertainty may be considered by default as unstable areas and the design becomes robust.

- Stages 1 and 2 are performed respectively. The designer has a stability map which is better than the one obtained with the classic deterministic approach (stable and unstable zones determined with the interval approach are $100 \%$ certain). The boundary between the stable and unstable zones is well-defined and the number of calculations is low.

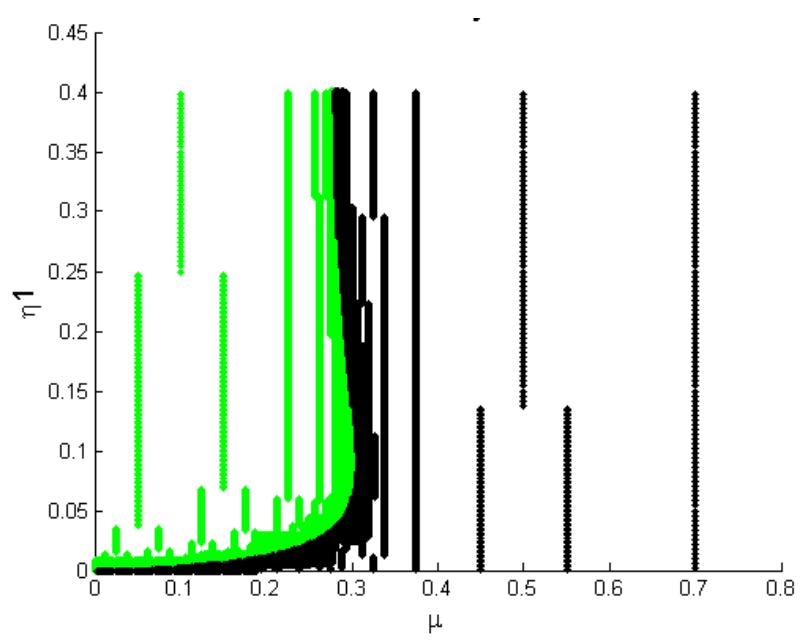

Fig. 8: Rough interval study and deterministic study: Stability map

Table 2: Number of calculations for the studies

\begin{tabular}{lc}
\hline Study & $\begin{array}{c}\text { Number of calcs. (\% reduction } \\
\text { w.r.to. deterministic study) }\end{array}$ \\
\hline $\begin{array}{l}\text { Deterministic study } \\
\text { Accurate study with intervals }\end{array}$ & 160801 \\
Rough study with intervals & $19304(25 \%)$ \\
$\begin{array}{l}\text { Rough study with intervals } \\
\text { and deterministic study }\end{array}$ & $13180(91 \%)$ \\
\hline
\end{tabular}

\section{Conclusions}

A new methodology to study the dynamic behaviour of friction systems, in particular the stability of brake systems, has been developed. This strategy allows the prediction of stable and unstable regions versus the variation of physical parameters. This method uses an interval analysis to reduce the number of studies. The efficiency and robustness of the method is demonstrated through numerical simulations. The interval approach helps to build a stability map with a low number of calculations. The stable areas are $100 \%$ certain. Therefore, the design becomes robust.

Finally, a deterministic approach allows the accurate determination of the boundary between the stable and unstable zones. For the interested reader, it may be noted the proposed algorithm works well in low dimensions due to the fact that the Routh-Hurwtz criterion is rather time consuming for mechanical systems with a large number of DoF dimensions. Future developments need to be considered for systems of large dimension by considering the Kharitonov Theorem [30] on the stability criterion for interval polynomials.

\section{ACKNOWLEDGEMENT:}

The authors gratefully acknowledge the financial support of the French National Research Agency through the Young Researcher program ANR-07-JCJC-0059-01CSD2. 


\section{REFERENCES:}

[1] R.A. Ibrahim. 1994. Friction-induced vibration, chatter, squeal and chaos: Part I - Mechanics of contact and friction, ASME Appl. Mech. Rev., 47(7), 209-226. doi:10.1115/1.3111079

[2] R.A. Ibrahim. 1994. Friction-induced vibration, chatter, squeal and chaos: Part II - Dynamics and modelling, ASME Appl. Mech. Rev., 47(7), 227-253. doi:10.1115/1.3111080

[3] F.P. Bowden and D. Tabor. 2001. The Friction and Lubrication of Solids, Second Ed. corrected, Oxford University Press, Clarendon Press, Oxford.

[4] J.T. Oden and J.A.C. Martins. 1985. Models and computational methods for dynamic friction phenomena, Computer Methods in Applied Mechanics and Engineering, 52, 527-634. doi:10.1016/00457825(85)90009-X

[5] H. Ouyang, J.E. Mottershead, M.P. Cartmell and M.I. Friswell. 1998. Friction-induced parametric resonances in discs: effect of a negative friction velocity relationship, $J$. Sound and Vibration, 209(2), 251-264. doi:10.1006/jsvi.1997.1261

[6] H. Ouyang, J.E. Mottershead, M.P. Cartmell and D.J. Brookfield. 1999. Friction-induced vibration of an elastic slider on a vibrating disc, Int. J. Mechanical Sciences, 41(3), 325-336. doi:10.1016/S0020-7403(98)00059-9

[7] H. Ouyang and J.E. Mottershead. 2001. Unstable travelling waves in the friction-induced vibration of discs, J. Sound and Vibration, 248(4), 768-779. doi:10.1006/jsvi.2001.3720

[8] M.R. North. 1972. A mechanism of disc brake squeal, Proc. $14^{\text {th }}$ FISITA Congress, Paper $1 / 9$. doi:10.1016/S0022-460X(02)01573-0

[9] N. Kinkaid, O. O'Reilly and P. Papadopoulos. 2003. Automotive disc brake squeal, J. Sound and Vibration, 267, 105-166.

[10] R.T. Spurr. 1961/1962. A theory of brake squeal. In: Proc. Automobile Division, Inst. Mech. Engineers, 1, 33-40.

[11] H.R. Mills. 1938/1939. Brake Squeal, Research Report 9000B and Research Report No. 9162B of the Inst. of Automobile Engineers.

[12] S.W.E. Earles and C.K. Lee. 1976. Instabilities arising from the frictional interaction of a pin-disc system resulting in noise generation, Trans. ASME J. Engineering for Industry , 98(1), 81-86. doi:10.1115/1.3438879

[13] N. Millner. 1978, An analysis of disc brake squeal, $S A E$ Paper 780332.

[14] B. Herve, J.J. Sinou, H. Mahe and L. Jezequel. 2008. Analysis of squeal noise and mode coupling instabilities including damping and gyroscopic effects, European $J$. $\begin{array}{llll}\text { Mechanics, } & \text { A/Solids } 27 & \text { (2), } & 141-160 .\end{array}$ doi:10.1016/j.euromechsol.2007.05.004

[15] J.J. Sinou, O. Dereure, G.B. Mazet, F. Thouverez and L. Jézéquel. 2006. Friction induced vibration for an aircraft brake system. Part I: experimental approach and stability analysis, Int. J. Mechanical Sciences, 48, 536-554. doi:10.1016/j.ijmecsci.2005.12.002
[16] J.J. Sinou and L. Jézéquel. 2007. Mode coupling instability in friction induced vibrations and its dependency on system parameters including damping, $J$. European Mechanics-A/Solids, 26, 106-122.

[17] J.J. Sinou, G. Fritz G and L. Jézéquel. 2007. The role of damping and definition of the robust damping factor for a self-exciting mechanism with constant friction, $J$. Vibration and Acoustics, 129, 297-306. doi:10.1115/1.2730536

[18] S. Lignon, J.J. Sinou and L. Jézéquel. 2006. Stability analysis and mu-synthesis control of brake systems. $J$. Sound and Vibration, 298, 1073-1087. doi:10.1016/j.jsv.2006.06.033

[19] S. Earles and P. Chambers. 1987. Disc brake squeal noise generation: Predicting its dependency on system parameters including damping, Int. J. Vehicle Design, 8, 538-552.

[20] J. Hultén. 1993. Drum brake squeal - A self-exciting mechanism with constant friction, SAE Paper 932965.

[21] J. Hultén. 1997. Friction phenomena related to drum brake squeal instabilities, Proc. ASME Design Engineering Technical Conf., ASME Paper DETC97/VIB-4161, Sacramento, California.

[22] N. Hoffmann and L. Gaul. 2003. Effects of damping on mode coupling instability in friction induced oscillations, Zeitschrift fur Angewandte Mathematik undMechanik, 83(8), 524-534.

[23] O.N. Kirillov, A.P. Seyranian. 2004. Stabilization and destabilization of a circulatory system by small velocitydependent forces, J. Sound and Vibration, 283(3-5), 781800. doi:10.1016/j.jsv.2004.05.020

[24] O.N. Kirillov. 2007. Destabilization paradox due to breaking the Hamiltonian and reversible symmetry, Int. J. Non-linear Mechanics, 42(1), 71-87. doi:10.1016/j.ijnonlinmec.2006.09.003

[25] R. Moore. 1966. Interval Analysis, Englewood Cliffs, NJ, Prentice-Hall.

[26] R. Moore. 1979. Methods and Applications of Interval Analysis, Philadelphia, SIAM.

[27] L. Jaulin, M. Kieffer, O. Didrit and E. Walter. 2001. Applied Interval Analysis, Springer, London.

[28] A. Rauh, M. Kletting, H. Aschemann and E.P. Hofer. 2004. Application of interval arithmetic simulation techniques to wasterwater treatment processes, Proc. $23^{r d}$ IASTED Int. Conf. Modeling, Identification and Control, Grindelwald, Switzerland, 287-293.

[29] M. Kletting, A. Rauh, H. Aschemann and E.P. Hofer. 2005. Consistency techniques for simulation of waste water treatment processes with uncertainties, Proc. IFAC World Congress, Prague, Czech Republic.

[30] V.L. Kharitonov. 1978. Asymptotic stability of an equilibrium position of a family of systems of differential equations, Differentsialnye uravneniya, 14, 2086-2088. (Russian). 